\title{
Patterns of drug use and HIV infection among adults in a nationally representative sample
}

\author{
Stephanie Shiau ${ }^{\mathrm{a}, \mathrm{b}}$, Stephen M. Arpadi ${ }^{\mathrm{a}, \mathrm{b}}$, Michael T. Yin ${ }^{\mathrm{c}}$, Silvia S. Martins ${ }^{\mathrm{b}, *}$ \\ a Gertrude H. Sergievsky Center, Columbia University Medical Center, 622 West $168^{\text {th }}$ Street, PH 19, New York, NY, United States \\ b Department of Epidemiology, Columbia University Mailman School of Public Health, 722 West 168th Street, New York, NY, United States \\ c Division of Infectious Diseases, Department of Medicine, Columbia University Medical Center, 630 West 168th Street, PH 8, New York, NY, United States
}

\section{H I G H L I G H T S}

- Little known on drug use patterns in people with HIV versus the general population

- Use of data from the National Survey on Drug Use and Health (2005-2014)

- Increased drug use in HIV-infected population in nationally representative sample

- Drug use can affect HIV disease progression, care, and adherence to treatment.

\section{A R T I C L E I N F O}

\section{Article history:}

Received 19 September 2016

Received in revised form 21 December 2016

Accepted 4 January 2017

Available online 7 January 2017

\section{Keywords:}

HIV

Aids

Drug use patterns

Representative sample

\begin{abstract}
A B S T R A C T
Background: Little is known about drug use patterns among people living with HIV in comparison to an uninfected group in the general population. The aim of this study was to investigate the association between legal and illegal drug use and HIV infection in a nationally representative sample of adults in the United States. Methods: Public use data files (2005-2014) from the National Survey on Drug Use and Health (NSDUH) were used. Respondents were asked whether a medical professional had ever told them that they had HIV/AIDS. Ever (lifetime), past-year, and past month use of cigarettes, alcohol, marijuana, cocaine, heroin, hallucinogens, inhalants, and nonmedical use of psychotherapeutics was assessed. Logistic regression was used to estimate adjusted odds ratios (aOR) of the relationship between drug use and HIV infection, adjusting for demographics. Results: Of 377,787 respondents age 18 and older, 548 (0.19\%) were categorized as HIV-infected. Ever use of cigarettes, tobacco, marijuana, cocaine, heroin, hallucinogens, inhalants, and psychotherapeutics was higher in HIVinfected individuals compared to HIV-uninfected individuals after adjustment for sex, age, race/ethnicity, education, total family income, and marital status. Past year and past month use was also higher for HIV-infected individuals for all substances aside from alcohol.

Conclusions: In a nationally representative sample, there are higher levels of drug use and DSM-IV dependence among the HIV-infected population compared to the HIV-uninfected population. This is of concern because drug use and dependence can impede engagement in HIV care and adherence to antiretroviral therapy.
\end{abstract}

(c) 2017 Elsevier Ltd. All rights reserved.

\section{Introduction}

Drug use and Human Immunodeficiency Virus (HIV) have been closely linked since the start of the HIV epidemic. In the United States, over 180,000 people with HIV who inject drugs have died over the course of the epidemic, accounting for $>25 \%$ of total deaths among people with Acquired Immune Deficiency Syndrome (AIDS) (Centers for

\footnotetext{
* Corresponding author at: Columbia University, Department of Epidemiology, 722 West 168th Street, Room 509, New York, NY, 10032, United States.

E-mail addresses: ss2568@columbia.edu (S. Shiau),sma2@columbia.edu (S.M. Arpadi), mty4@columbia.edu (M.T. Yin), ssm2183@columbia.edu (S.S. Martins).
}

Disease Control and Prevention, 2015). Drug use is a well-established risk factor of HIV infection. Of an estimated 44,073 people newly diagnosed with HIV infection in the United States in 2014, approximately $6 \%$ were attributed to injection drug use and an additional $3 \%$ were due to injection drug use in combination with male-to-male sexual contact (Centers for Disease Control and Prevention, 2014). Recently, an outbreak in Indiana of 181 new cases of HIV infection in a community of 4200 people over 12 months in 2014-2015 was linked to injection use of prescription opiates (Conrad et al., 2015). Although injection drug use can directly lead to HIV transmission via the sharing of drug preparation or injection equipment, drinking, smoking, or inhaling drugs such as alcohol, cocaine, and methamphetamine can further 
increase HIV infection risk by adversely affect decision making and increasing risky sexual behavior (Baggaley, Boily, White, \& Alary, 2006; Hudgins, McCusker, \& Stoddard, 1995; Molitor, Truax, Ruiz, \& Sun, 1998; Purcell, Parsons, Halkitis, Mizuno, \& Woods, 2001).

In addition to increasing the risk of HIV transmission, for those already infected with HIV, drug use poses several health challenges. Some evidence indicates substance use can alter HIV disease progression by immune modulation and increasing HIV viral load (Kapadia, Vlahov, Donahoe, \& Friedland, 2005). In addition, drug use can negatively affect adherence to HIV treatment and engagement with health care (Azar, Springer, Meyer, \& Altice, 2010; Hinkin et al., 2007), leading to adverse clinical outcomes. As a result, the management of HIV in drug users requires a careful consideration of many factors, including comorbid medical and mental health conditions, medication side effects and toxicities, and drug interactions.

Existing studies on HIV and drug use in the United States primarily report on HIV infection rates among injection drug users in comparison to non-injection drug users (Spiller, Broz, Wejnert, Nerlander, \& Paz-Bailey, 2015; Strathdee \& Stockman, 2010) or on the prevalence of drug use among a population with HIV (Galvan et al., 2002; Ompad et al., 2016). Few studies, however, have specifically compared drug use patterns among people living with HIV to people without HIV in the general United States population. Furthermore, it is unclear if the burden of HIV and substance use varies across sub-groups of the population, such as young adults or racial/ethnic minorities. Carefully studying these patterns may identify specific groups of drug users to target for HIV prevention, as well as drug users with HIV to target for substance abuse prevention or treatment as well as HIV care. In this study, we investigated the association between legal and illegal drug use and HIV infection in a nationally representative sample of adults in the United States. In addition, we assessed the association between HIV and illegal drug use across age, race/ethnicity, and sex groups.

\section{Materials and methods}

\subsection{Data source}

We used data from the National Survey on Drug Use and Health (NSDUH), an annual national survey sponsored by the Substance Abuse and Mental Health Services Administration (SAMHSA) that evaluates the use of legal and illegal drugs in the noninstitutionalized United States citizen population, age 12 and older (Substance Abuse and Mental Health Services Administration, 2005-2014). Each year, households are randomly selected to participate in the NSDUH and data are collected using questionnaires administered through face-to-face interviews. Sampling weights were created to account for probability of selection into the sample, correct for nonresponse, and adjust for known population distributions (Substance Abuse and Mental Health Services Administration, 2012). Public use data files from 2005 to 2014 were combined for this analysis. From a total of 560,099 observations from all years, we excluded 176,879 participants under the age of 18 and an additional 5433 with missing/unknown HIV infection status (only $0.97 \%$ of the total sample), leaving us with a total of 377,787 participants for analysis.

\subsection{Measures}

The following socio-demographic characteristics were considered in this analysis: sex, age, race/ethnicity, education, total family income, and marital status. Race/ethnicity included the following categories: non-Hispanic White, non-Hispanic Black, Hispanic, and Other (Native American, Native Hawaiian or Pacific Islander, Asian, and more than one race). New questions asking about 20 lifetime health conditions have been added to the NSDUH questionnaire since 2005. Respondents were asked whether doctors or other medical professionals had ever told them that they had HIV/AIDS and were categorized as HIV-infected if they responded yes. Ever (lifetime) use, past-year use, past month use, was assessed with a dichotomous (yes/no) variable for a number of substances including cigarettes, alcohol, marijuana, cocaine, heroin, hallucinogens, inhalants, and non-medical psychotherapeutics (analgesics including prescription opioids, stimulants including methamphetamine, sedatives, and tranquilizers). Past-year DSM-IV-TR dependence (met three or more of seven DSM-IV dependence criteria) was also assessed (American Psychiatric Association, 2000). Any illegal drug use and DSM-IV dependence was also assessed.

\subsection{Statistical analyses}

First, descriptive characteristics of the sample were calculated stratified by HIV-infection using weighted cross-tabulations. Next, drug use characteristics were compared between HIV-infection groups using weighted-cross tabulations. Chi-squared tests were used to determine differences in characteristics by HIV infection group. Weighted logistic regression was then used to estimate unadjusted odds ratios (OR) and 95\% confidence intervals of drug use comparing HIV-infected participants to HIV-uninfected participants. Multivariable multinomial logistic regression was then used to generate adjusted odds ratios (aOR) to evaluate the relationship between drug use and HIV infection, adjusting for potential confounders including sex, age, race/ethnicity, education, total family income, and marital status. Finally, to better understand the burden of substance use and HIV across sub-groups, we compared the prevalence of past year illegal drug use between HIV-infected and uninfected participants within sub-groups, including sex (male vs. female), age (18-25 vs. $26-34$ vs. $35-49$ vs. 50 ), and race/ethnicity (non-Hispanic White, non-Hispanic Black, and Hispanic) using Chisquared tests. The Other race/ethnicity group was excluded due to small numbers. Weighted logistic regression was used to calculate the OR and 95\% confidence intervals of illegal drug use comparing HIV-infected participants to HIV-uninfected participants within sub-groups, adjusted for other demographic variables. Data were analyzed with SAS 9.4 (Cary, NC, USA) and adjusted for weighting and clustering to reflect the complex design of the NSDUH sample. All $p$-values are 2-tailed and $p$-values $<0.05$ were considered statistically significant.

\section{Results}

\subsection{Demographic characteristics by HIV infection group}

Data on characteristics of 377,787 respondents age 18 and older are presented in Table 1, stratified by HIV infection group. A total of 548 ( $0.19 \%$ weighted) adults were categorized as HIV-infected. Sex, age, race/ethnicity, family income, and marital status were significantly different between the HIV-uninfected and HIV-infected group. While sex was balanced in the HIV-uninfected group (48\% male), $80 \%$ of the HIVinfected group was male $(p<0.0001)$. A higher proportion of the HIVinfected group was 35-49 years of age compared to the HIV-uninfected group ( 41.4 vs. $27.5 \%, p<0.0001$ ). In addition, a greater proportion of the HIV-infected group was non-Hispanic Black (29.2 vs. $11.4 \%$, $p<0.0001)$ and Hispanic (18.8 vs. $13.9 \%, p<0.0001)$ compared to the HIV-uninfected group. Forty percent of the HIV-infected group had a total family income $<\$ 19,000$ compared to $18.3 \%$ in the HIV-uninfected group. A larger proportion of the HIV-infected group had never been married compared to the HIV-uninfected group (68.1 vs. $26.2 \%$, $p<0.0001)$.

\subsection{Drug use characteristics by HIV infection group}

A higher proportion of the HIV-infected group reported ever use of cigarettes, tobacco, alcohol, marijuana, cocaine, heroin, hallucinogens, inhalants, and non-medical psychotherapeutics, and any illegal drug use compared to the HIV-uninfected group (Table 2). Three-quarters of the HIV-infected participants reported ever using marijuana 
Table 1

Selected characteristics of participants living without and with HIV/AIDS age 18 and older $(N=377,787)$. Percentages are weighted to be nationally representative. Data from the 2005 to 2014 National Survey on Drug Use and Health, United States.

\begin{tabular}{|c|c|c|c|c|c|c|c|}
\hline \multirow[t]{2}{*}{ Characteristics } & \multicolumn{2}{|c|}{ Total sample $N=377.787$} & \multicolumn{2}{|c|}{ HIV-uninfected $N=377.239$} & \multicolumn{2}{|c|}{ HIV-infected $N=548$} & \multirow[b]{2}{*}{$p$-Value } \\
\hline & $N$ & $\mathrm{Wt} \%$ (SE) & $N$ & $\mathrm{Wt} \%(\mathrm{SE})$ & $N$ & $\mathrm{Wt} \%(\mathrm{SE})$ & \\
\hline \multicolumn{8}{|l|}{ Sex } \\
\hline Male & 175,197 & $48.1(0.13)$ & 174,790 & $48.0(0.13)$ & 407 & $80.1(2.2)$ & \multirow[t]{2}{*}{$<0.0001$} \\
\hline Female & 202,590 & $51.9(0.13)$ & 202,449 & $52.0(0.13)$ & 141 & $19.9(2.2)$ & \\
\hline \multicolumn{8}{|l|}{ Age } \\
\hline $18-25$ & 178,024 & $14.6(0.09)$ & 177,898 & $14.7(0.09)$ & 126 & $5.7(0.7)$ & \multirow[t]{4}{*}{$<0.0001$} \\
\hline $26-34$ & 58,034 & $15.8(0.09)$ & 57,950 & $15.8(0.09)$ & 84 & $15.6(2.0)$ & \\
\hline $35-49$ & 80,984 & $27.5(0.13)$ & 80,743 & $27.5(0.13)$ & 241 & $41.4(2.9)$ & \\
\hline $50+$ & 60,745 & $42.0(0.18)$ & 60,648 & $42.1(0.18)$ & 97 & $37.3(2.8)$ & \\
\hline \multicolumn{8}{|l|}{ Race/ethnicity } \\
\hline Non-Hispanic White & 241,855 & $58.0(0.19)$ & 241,598 & $68.1(0.18)$ & 257 & $51.5(3.7)$ & \multirow[t]{4}{*}{$<0.0001$} \\
\hline Non-Hispanic Black & 46,083 & $11.5(0.13)$ & 45,896 & $11.4(0.13)$ & 187 & $29.2(3.3)$ & \\
\hline Hispanic & 57,964 & $13.9(0.13)$ & 57,870 & $13.9(0.13)$ & 94 & $18.8(2.6)$ & \\
\hline Other & 31,885 & $6.6(0.10)$ & 31,875 & $6.6(0.10)$ & 10 & $0.5(0.4)$ & \\
\hline \multicolumn{8}{|l|}{ Education } \\
\hline Less than high school & 294,313 & $71.2(0.18)$ & 293,897 & $71.2(0.18)$ & 416 & $67.0(3.6)$ & \multirow[t]{2}{*}{0.226} \\
\hline High school graduate or more & 83,474 & $28.8(0.18)$ & 83,342 & $28.7(0.18)$ & 132 & $33.0(3.6)$ & \\
\hline \multicolumn{7}{|l|}{ Total family income } & \multirow[t]{5}{*}{$<0.0001$} \\
\hline$<\$ 19,000$ & 94,937 & $18.4(0.14)$ & 94,707 & $18.3(0.14)$ & 230 & $40.1(3.6)$ & \\
\hline$\$ 20,000-39,000$ & 130,384 & $32.9(0.14)$ & 130,214 & $32.9(0.140)$ & 170 & $29.7(2.7)$ & \\
\hline$\$ 40,000-74,000$ & 60,878 & $17.4(0.13)$ & 60,820 & $17.5(0.13)$ & 58 & $11.2(2.2)$ & \\
\hline$\$ 75,000+$ & 91,588 & $31.4(0.20)$ & 91,498 & $31.4(0.20)$ & 90 & $19.1(3.2)$ & \\
\hline \multicolumn{8}{|l|}{ Marital status } \\
\hline Currently married & 139,779 & $54.0(0.17)$ & 139,722 & $54.0(0.17)$ & 57 & $14.1(2.3)$ & \multirow[t]{3}{*}{$<0.0001$} \\
\hline Previously married & 45,350 & $19.8(0.12)$ & 45,248 & $19.8(0.12)$ & 102 & $17.8(2.2)$ & \\
\hline Never married & 192,658 & $26.3(0.13)$ & 192,269 & $26.2(0.13)$ & 389 & $68.1(2.8)$ & \\
\hline
\end{tabular}

compared to $44.5 \%$ of the HIV-uninfected participants. Close to half of the HIV-infected participants reported ever cocaine use (57.6\%), ever hallucinogen use (49.2\%), ever inhalant use (42.7\%), and ever non-medical psychotherapeutic use (47.4\%). Over $80 \%$ of the HIV-infected participants reported ever using illegal drugs compared to just under $50 \%$ of the HIV-uninfected participants. Past year and past month use of all drugs aside from alcohol was significantly higher in the HIV-infected group compared to the HIV-uninfected group. Close to half (46.1\%) of the HIV-infected participants reported any past year illegal drug use compared to $14.7 \%$ of the HIV-uninfected participants ( $p<0.0001)$. A third (36\%) of the HIV-infected participants reported any past month illegal drug use compared to $8.6 \%$ of the HIV-uninfected participants $(p<0.0001)$.

\subsection{HIV and drug use: logistic regression results}

To adjust for potential confounding of the association between HIV and drug use, we conducted weighted multinomial logistic regression analyses adjusted for sex, age, race/ethnicity, education, total family income, and marital status (Table 3). In adjusted analyses, ever use of all drugs was higher in HIV-infected participants compared to HIV-uninfected participants, including cigarettes (aOR: 1.63, 95\% CI: 1.22, 2.17), tobacco (aOR: 1.50, 95\% CI: 1.10, 2.05), alcohol (aOR: 2.47, 95\% CI: $1.40,4.33$ ), marijuana (aOR: $3.58,95 \% \mathrm{CI}: 2.75,4.66)$, cocaine (aOR: 5.49 , 95\% CI: 4.10, 7.35), heroin (OR: $3.84,95 \% \mathrm{CI}: 2.47,5.98$ ), hallucinogens (aOR: 4.55, 95\% CI: 3.37, 6.14), inhalants (aOR: 6.85, 95\% CI: 5.12, 9.15), psychotherapeutics (aOR: 3.11, 95\% CI: 2.31, 4.18). Ever use of any illegal drug use was $>4$ times higher in HIV-infected participants compared to HIV-uninfected participants (aOR: 4.62, 95\% CI: 3.39, 6.29). Past year use of cigarettes (aOR: $2.15,95 \% \mathrm{CI}: 1.67,2.77$ ), tobacco (aOR: 1.59, 95\% CI: 1.24, 2.05), marijuana (aOR: 3.16, 95\% CI: 2.41, 4.16), cocaine (aOR: 4.56, 95\% CI: 3.16, 6.58), heroin (aOR: 3.64, 95\% CI: 1.08, 12.25), hallucinogens (aOR: $3.76,95 \% \mathrm{CI}: 2.28,6.22$ ), inhalants (aOR: 14.69, 95\% CI: 9.90, 21.79), and non-medical psychotherapeutics (aOR: $2.73,95 \% \mathrm{CI}: 1.93,3.86$ ) was significantly higher among those with HIV-infection compared to those without HIV-infection, after adjustment for sex, age, race/ethnicity, education, total family income, and marital status. Results were very similar for past month use of cigarettes
(aOR: 2.09, 95\% CI: 1.63, 2.68), tobacco (aOR: 1.63, 95\% CI: 1.29, 2.06), marijuana (aOR: 3.31, 95\% CI: 2.49, 4.40), cocaine (aOR: 5.27, 95\% CI: $3.25,8.55$ ), heroin (aOR: 6.36, 95\% CI: 1.80, 22.44), hallucinogens (aOR: $4.04,95 \%$ CI: $1.86,8.80$ ), inhalants (aOR: $16.94,95 \%$ CI: 10.22 , 28.06), and non-medical psychotherapeutics (aOR: 3.09, 95\% CI: 1.95, 4.90). Past year DSM-IV dependence of any illicit drug use was 4 times higher in HIV-infected participants compared to HIV-uninfected participants (aOR 4.34, 95\% CI: 2.87, 6.57).

\subsection{Past year illegal drug use in sub-groups}

The prevalence of past-year illegal drug use was greater in males with and without HIV-infection than females (Table 4), with half of all males with HIV-infection using illegal drugs in the past year. The association between HIV and past year illegal drug use was higher in males (OR: $4.19,95 \% \mathrm{CI}: 3.16,5.56$ ) compared to females (OR: $2.76,95 \% \mathrm{CI}$ : $1.64,4.66)$. Across all age groups, the prevalence of past-year illegal drug use was higher in HIV-infected participants compared to HIV-uninfected participants (Table 4). The prevalence decreased from $62.7 \%$ among the youngest HIV-infected group to $36.4 \%$ in the oldest HIV-infected group. In weighted logistic regression models, the odds of past year illegal drug use was 2.76 (95\% CI: 1.66, 4.58), 3.33 (95\% CI: 1.82 , 6.09), 3.63 (95\% CI: 2.47, 5.35), and 4.83 (95\% CI: $2.71,8.58$ ) times higher in HIV-infected adults than HIV-uninfected adults for those 1825 years, $26-34$ years, $35-49$ years, and $>50$ years, respectively. The prevalence of past-year illegal drug use was significantly higher in HIV-infected participants compared to HIV-uninfected participants in all race/ethnicity groups (Table 4), and similar between race/ethnicity groups. The odds of past year illegal drug use was similar across race/ ethnicity groups, including among non-Hispanic Whites (OR: 4.37, 95\% CI: 2.96, 6.44), non-Hispanic Blacks (OR: 3.35, 95\% CI: 2.13, 5.26), and Hispanics (OR: 3.94, 95\% CI: 2.05, 7.57).

\section{Discussion}

Our study compares drug use characteristics in HIV-infected individuals to HIV-uninfected individuals in a nationally representative sample. We found higher levels of reported ever drug use among HIV-infected 
Table 2

Drug use characteristics of participants living without and with HIV/AIDS age 18 and older $(N=377,787)$. Percentages are weighted to be nationally representative. Data from the 2005 to 2014 National Survey on Drug Use and Health, United States.

\begin{tabular}{|c|c|c|c|c|c|c|c|}
\hline \multirow[t]{2}{*}{ Characteristics } & \multicolumn{2}{|c|}{ Total sample $N=377.787$} & \multicolumn{2}{|c|}{ HIV-uninfected $N=377.239$} & \multicolumn{2}{|c|}{ HIV-infected $N=548$} & \multirow[b]{2}{*}{$p$-Value } \\
\hline & $N$ & $\mathrm{Wt} \%(\mathrm{SE})$ & $N$ & $\mathrm{Wt} \%(\mathrm{SE})$ & $N$ & $\mathrm{Wt} \%$ (SE) & \\
\hline \multicolumn{8}{|l|}{ Ever } \\
\hline Cigarette & 254,248 & $68.7(0.12)$ & 253,813 & $68.7(0.12)$ & 435 & $79.4(2.4)$ & $<0.0001$ \\
\hline Any tobacco & 274,301 & $73.4(0.11)$ & 273,850 & $73.4(0.11)$ & 451 & $83.3(2.2)$ & 0.0001 \\
\hline Alcohol & 330,728 & $87.5(0.10)$ & 330,215 & $87.5(0.10)$ & 513 & $94.6(1.5)$ & 0.001 \\
\hline Marijuana & 189,121 & $44.6(0.14)$ & 188,715 & $44.5(0.14)$ & 406 & $76.7(2.4)$ & $<0.0001$ \\
\hline Cocaine & 60,337 & $15.9(0.10)$ & 60,069 & $15.9(0.10)$ & 268 & $57.6(3.0)$ & $<0.0001$ \\
\hline Heroin & 7230 & $1.78(0.03)$ & 7175 & $1.76(0.03)$ & 55 & $11.4(2.1)$ & $<0.0001$ \\
\hline Hallucinogens & 68,278 & $15.8(0.09)$ & 68,050 & $15.7(0.09)$ & 228 & $49.2(3.1)$ & $<0.0001$ \\
\hline Inhalants & 37,994 & $8.7(0.08)$ & 37,790 & $8.6(0.08)$ & 204 & $42.7(2.9)$ & $<0.0001$ \\
\hline Psychotherapeutics & 96,140 & $21.6(0.09)$ & 95,890 & $21.5(0.09)$ & 250 & $47.4(3.3)$ & $<0.0001$ \\
\hline Any illegal drug use & 209,431 & $49.6(0.13)$ & 208,981 & $49.6(0.13)$ & 450 & $83.8(2.1)$ & $<0.0001$ \\
\hline \multicolumn{8}{|l|}{ Past year } \\
\hline Cigarette & 139,303 & $28.5(0.13)$ & 139,001 & $28.4(0.13)$ & 302 & $50.9(3.0)$ & $<0.0001$ \\
\hline Any tobacco & 164,607 & $34.5(0.13)$ & 164,286 & $34.4(0.13)$ & 321 & $53.0(3.0)$ & $<0.0001$ \\
\hline Alcohol & 283,089 & $70.5(0.15)$ & 282,675 & $70.5(0.15)$ & 414 & $75.5(2.9)$ & 0.098 \\
\hline Marijuana & 72,425 & $11.2(0.08)$ & 72,215 & $11.1(0.08)$ & 210 & $34.2(2.9)$ & $<0.0001$ \\
\hline Cocaine & 12,849 & $2.0(0.03)$ & 12,769 & $2.0(0.03)$ & 80 & $12.9(2.0)$ & $<0.0001$ \\
\hline Heroin & 1574 & $0.24(0.01)$ & 1564 & $0.24(0.01)$ & 10 & $1.7(1.0)$ & 0.0002 \\
\hline Hallucinogens & 13,346 & $1.5(0.02)$ & 13,303 & $1.5(0.02)$ & 43 & $5.3(1.3)$ & $<0.0001$ \\
\hline Inhalants & 3362 & $0.46(0.02)$ & 3298 & $0.44(0.02)$ & 64 & $9.9(1.6)$ & $<0.0001$ \\
\hline Psychotherapeutics & 35,759 & $6.03(0.06)$ & 35,655 & $6.01(0.06)$ & 104 & $16.1(2.3)$ & $<0.0001$ \\
\hline Any illegal drug use & 89,037 & $14.7(0.10)$ & 88,753 & $14.7(0.10)$ & 284 & $46.1(2.9)$ & $<0.0001$ \\
\hline \multicolumn{8}{|l|}{ Past month } \\
\hline Cigarette & 116,544 & $24.6(0.13)$ & 116,273 & $24.5(0.13)$ & 271 & $45.7(3.1)$ & $<0.0001$ \\
\hline Any tobacco & 136,543 & $29.3(0.13)$ & 136,258 & $29.3(0.13)$ & 285 & $47.3(3.1)$ & $<0.0001$ \\
\hline Alcohol & 222,369 & $55.9(0.15)$ & 222,024 & $55.8(0.15)$ & 345 & $61.9(3.1)$ & 0.056 \\
\hline Marijuana & 43,769 & $6.8(0.06)$ & 43,618 & $6.7(0.06)$ & 151 & $24.9(2.5)$ & $<0.0001$ \\
\hline Cocaine & 4159 & $0.74(0.02)$ & 4121 & $0.73(0.02)$ & 38 & $7.3(1.6)$ & $<0.0001$ \\
\hline Heroin & 648 & $0.11(0.007)$ & 642 & $0.11(0.006)$ & 6 & $1.5(1.0)$ & $<0.0001$ \\
\hline Hallucinogens & 3391 & $0.40(0.01)$ & 3376 & $0.40(0.01)$ & 15 & $1.8(0.67)$ & $<0.0001$ \\
\hline Inhalants & 849 & $0.15(0.01)$ & 815 & $0.14(0.01)$ & 34 & $6.0(1.4)$ & $<0.0001$ \\
\hline Psychotherapeutics & 14,769 & $2.6(0.04)$ & 14,715 & $2.5(0.04)$ & 54 & $8.8(1.8)$ & $<0.0001$ \\
\hline Any illegal drug use & 52,693 & $8.6(0.07)$ & 52,489 & $8.6(0.07)$ & 204 & $36.0(2.9)$ & $<0.0001$ \\
\hline \multicolumn{8}{|c|}{ Past year DSM-IV dependence } \\
\hline Alcohol & 18,861 & $3.4(0.04)$ & 18,817 & $3.4(0.04)$ & 44 & $6.1(1.5)$ & 0.018 \\
\hline Marijuana & 7733 & $0.96(0.02)$ & 7708 & $0.95(0.02)$ & 25 & $2.9(0.8)$ & $<0.0001$ \\
\hline Cocaine & 1838 & $0.35(0.01)$ & 1809 & $0.34(0.01)$ & 29 & $4.6(1.3)$ & $<0.0001$ \\
\hline Heroin & 882 & $0.14(0.008)$ & 876 & $0.14(0.008)$ & 6 & $1.5(1.0)$ & $<0.0001$ \\
\hline Hallucinogens & 360 & $0.04(0.003)$ & 356 & $0.04(0.003)$ & 4 & $0.24(0.14)$ & 0.0002 \\
\hline Inhalants & 75 & $0.01(0.002)$ & 71 & $0.01(0.002)$ & 4 & $0.59(0.35)$ & $<0.0001$ \\
\hline Psychotherapeutics & 4121 & $0.64(0.02)$ & 4102 & $0.63(0.02)$ & 19 & $2.8(0.8)$ & $<0.0001$ \\
\hline Any illicit drug & 12,812 & $1.8(0.03)$ & 12,745 & $1.8(0.03)$ & 67 & $10.2(1.8)$ & $<0.0001$ \\
\hline
\end{tabular}

adults compared to HIV-uninfected adults in the United States. For past year and past month use, the HIV-infected group consistently reported higher use of all substances other than alcohol compared to the HIV-uninfected group, even after adjustment for demographic variables. This study is of particular importance as few studies have estimated drug usage among HIV-infected populations in comparison to the general population. The majority of epidemiologic studies report on HIV infection rates among injection drug users in comparison to non-injection drug users (Spiller et al., 2015; Strathdee \& Stockman, 2010) or on the prevalence of drug use among a population with HIV (Galvan et al., 2002; Ompad et al., 2016).

Our finding of both higher lifetime and recent drug use in HIV-infected adults versus uninfected adults is of great concern. Drug use can affect HIV disease progression, impede engagement in care, and affect adherence to antiretroviral therapy. Specific drugs have been shown to affect disease progression and quality of life even among those with suppressed virus on treatment. A study of suppressed HIV-infected individuals found users of methamphetamine to have greater CD4 T-cell and CD8 T-cell proliferation as well as exhaustion of the immune system (Massanella et al., 2015). In infected adults receiving care, those with current illicit drug use and polysubstance use have decreased mental and physical health-related quality of life (Korthuis et al., 2008). Unfortunately, drug use can negatively affect adherence to HIV treatment and engagement with health care (Arnsten et al., 2002; Azar et al., 2010;
Hinkin et al., 2007; Nolan et al., 2011). Life expectancy consequences of HIV and drug use may also exist (Helleberg et al., 2013; Marcus et al., 2016; Samji et al., 2013). A recent study of HIV-infected adults in the Kaiser Permanente cohort in 1996-2011 and matched HIV-uninfected adults which estimated a life expectancy gap for the HIV-infected adults, particularly among those who use injection drugs (Marcus et al., 2016). Treatment considerations are also needed for HIV-infected drug users, especially around drug interactions when treating both drug addiction and HIV-infection. For example, the concentration of methadone, used to treat opioid addictions and reduce pain, is decreased in blood when used in combination with certain antiretroviral regimens (McCance-Katz, 2005).

Apart from adverse effects on an individual's health, substance use among people living with HIV has implications for continued spread of HIV through risky sexual behavior. Drug use is a strong predictor of poor adherence and, consequently, failure to maintain viral suppression (Arnsten et al., 2002; Cropsey et al., 2016). Increases in viral load have been shown to be associated with an increased risk of HIV transmission (Attia, Egger, Muller, Zwahlen, \& Low, 2009; Gray et al., 2001).

We did not find past year and past month alcohol use to be significantly different between the HIV-infected and HIV-uninfected groups. This finding is consistent with a national probability survey of HIV-infected adults receiving medical care in the United States which estimated $53.4 \%$ of the population of HIV-infected persons to have consumed 
Table 3

Unadjusted and adjusted weighted binary logistic regression model for the association between HIV status and drug use.

Data from the 2005 to 2014 National Survey on Drug Use and Health, United States.

\begin{tabular}{|c|c|c|}
\hline Characteristics & $\begin{array}{l}\text { Unadjusted OR }(95 \% \mathrm{CI}) \\
(\mathrm{HIV}+\mathrm{vs} . \mathrm{HIV}-)\end{array}$ & $\begin{array}{l}\text { *Adjusted OR }(95 \% \mathrm{CI}) \\
(\mathrm{HIV}+\text { vs. HIV - ) }\end{array}$ \\
\hline \multicolumn{3}{|l|}{ Ever } \\
\hline Cigarette & $1.76(1.32,2.34)$ & $1.63(1.22,2.17)$ \\
\hline Any tobacco & $1.80(1.33,2.44)$ & $1.50(1.10,2.05)$ \\
\hline Alcohol & $2.51(1.42,4.44)$ & $2.47(1.40,4.33)$ \\
\hline Marijuana & $4.09(3.15,5.33)$ & $3.58(2.75,4.66)$ \\
\hline Cocaine & $7.19(5.64,9.16)$ & $5.49(4.10,7.35)$ \\
\hline Heroin & $7.21(4.75,10.95)$ & $3.84(2.47,5.98)$ \\
\hline Hallucinogens & $5.18(4.07,6.61)$ & $4.55(3.37,6.14)$ \\
\hline Inhalants & $7.92(6.27,10.00)$ & $6.85(5.12,9.15)$ \\
\hline Psychotherapeutics & $3.29(2.53,4.26)$ & $3.11(2.31,4.18)$ \\
\hline Any illegal drug use & $5.26(3.89,7.12)$ & $4.62(3.39,6.29)$ \\
\hline \multicolumn{3}{|l|}{ Past year } \\
\hline Cigarette & $2.61(2.06,3.30)$ & $2.15(1.67,2.77)$ \\
\hline Any tobacco & $2.15(1.70,2.72)$ & $1.59(1.24,2.05)$ \\
\hline Alcohol & $1.29(0.95,1.74)$ & $1.33(0.93,1.90)$ \\
\hline Marijuana & $4.15(3.24,5.32)$ & $3.16(2.41,4.16)$ \\
\hline Cocaine & $7.24(5.13,10.21)$ & $4.56(3.16,6.58)$ \\
\hline Heroin & $7.17(2.17,23.63)$ & $3.64(1.08,12.25)$ \\
\hline Hallucinogens & $3.60(2.15,6.02)$ & $3.76(2.28,6.22)$ \\
\hline Inhalants & $24.75(16.99,36.06)$ & $14.69(9.90,21.79)$ \\
\hline Psychotherapeutics & $3.01(2.16,4.18)$ & $2.73(1.93,3.86)$ \\
\hline Any illegal drug use & $4.98(3.96,6.26)$ & $3.98(3.07,5.16)$ \\
\hline \multicolumn{3}{|l|}{ Past month } \\
\hline Cigarette & $2.59(2.03,3.31)$ & $2.09(1.63,2.68)$ \\
\hline Any tobacco & $2.17(1.71,2.76)$ & $1.63(1.29,2.06)$ \\
\hline Alcohol & $1.28(0.99,1.66)$ & $1.21(0.90,1.63)$ \\
\hline Marijuana & $4.60(3.54,5.99)$ & $3.31(2.49,4.40)$ \\
\hline Cocaine & $10.70(6.68,17.15)$ & $5.27(3.25,8.55)$ \\
\hline Heroin & $14.54(4.06,52.03)$ & $6.36(1.80,22.44)$ \\
\hline Hallucinogens & $4.59(2.16,9.76)$ & $4.04(1.86,8.80)$ \\
\hline Inhalants & $45.40(27.94,73.77)$ & $16.94(10.22,28.06)$ \\
\hline Psychotherapeutics & $3.70(2.39,5.71)$ & $3.09(1.95,4.90)$ \\
\hline Any illegal drug use & $5.98(4.68,7.64)$ & $4.45(3.39,5.82)$ \\
\hline \multicolumn{3}{|c|}{ Past year DSM-IV dependence } \\
\hline Alcohol & $1.86(1.10,3.12)$ & $1.17(0.69,1.99)$ \\
\hline Marijuana & $3.12(1.80,5.41)$ & $2.31(1.32,4.06)$ \\
\hline Cocaine & $14.00(7.88,24.86)$ & $6.01(3.22,11.21)$ \\
\hline Heroin & $11.02(2.97,40.91)$ & $5.19(1.39,19.31)$ \\
\hline Hallucinogens & $6.32(2.09,19.17)$ & $5.74(1.84,17.94)$ \\
\hline Inhalants & $70.66(22.29,224.02)$ & $64.29(14.60,283.20)$ \\
\hline Psychotherapeutics & $4.43(2.50,7.87)$ & $3.95(2.17,7.20)$ \\
\hline Any illicit drug & $6.14(4.16,9.07)$ & $4.34(2.87,6.57)$ \\
\hline
\end{tabular}

* Adjusted for sex (reference $=$ female), age (reference $=50+$ ), race/ethnicity (reference $=$ non-Hispanic white), education (reference $=$ high school graduate or more), total family income (reference $=\$ 75,000+$ ), marital status (reference $=$ currently married).

Table 4

Past year illegal drug use of participants living without and with HIV/AIDS age 18 and older ( $N=377,787)$ stratified by sex, age, and race/ethnicity. Percentages are weighted to be nationally representative.

Data from the 2005 to 2014 National Survey on Drug Use and Health, United States.

\begin{tabular}{|c|c|c|c|c|c|}
\hline \multirow[t]{2}{*}{ Strata } & \multicolumn{2}{|c|}{$\begin{array}{l}\text { HIV-uninfected } \\
N=377.239\end{array}$} & \multicolumn{2}{|c|}{$\begin{array}{l}\text { HIV-infected } \\
N=548\end{array}$} & \multirow[b]{2}{*}{$p$-Value } \\
\hline & $N$ & $\mathrm{Wt} \%$ (SE) & $N$ & Wt\% (SE) & \\
\hline \multicolumn{6}{|l|}{ Sex } \\
\hline Male & 48,218 & $17.7(0.2)$ & 231 & $50.6(3.4)$ & $<0.0001$ \\
\hline Female & 40,535 & $11.9(0.1)$ & 53 & $28.2(5.0)$ & $<0.0001$ \\
\hline \multicolumn{6}{|l|}{ Age } \\
\hline $18-25$ & 61,236 & $35.1(0.2)$ & 79 & $62.7(6.2)$ & $<0.0001$ \\
\hline $26-34$ & 12,699 & $22.0(0.3)$ & 53 & $57.9(7.9)$ & $<0.0001$ \\
\hline $35-49$ & 11,054 & $13.1(0.1)$ & 114 & $48.2(4.6)$ & $<0.0001$ \\
\hline $50+$ & 3764 & $5.8(0.1)$ & 38 & $36.4(6.5)$ & $<0.0001$ \\
\hline \multicolumn{6}{|l|}{ Race/ethnicity } \\
\hline Non-Hispanic White & 58,238 & $14.9(0.1)$ & 142 & $49.8(4.4)$ & $<0.0001$ \\
\hline Non-Hispanic Black & 11,405 & $16.4(0.3)$ & 86 & $41.9(5.4)$ & $<0.0001$ \\
\hline Hispanic & 11,626 & $13.5(0.2)$ & 47 & $43.2(7.3)$ & $<0.0001$ \\
\hline
\end{tabular}

alcohol in the past month, similar to a general population estimate of 53.5\% reported by the Substance Abuse and Mental Health Services Administration (SAMHSA) (Galvan et al., 2002). Despite no elevated alcohol use in the HIV-infected group, alcohol use may be uniquely harmful in this population. A recent analysis of data from the Veterans Aging Cohort study found that HIV-infected adults had a higher risk of mortality or physiological injury in comparison to HIV-uninfected individuals, even among lower alcohol consumption levels and among individuals with suppressed viral load (Justice et al., 2016).

Our data suggests the combination of HIV and drug use may affect sub-groups of the population. Consistent with other national estimates indicating that HIV has a disproportionate impact on racial and ethnic minorities in the United States (Pellowski, Kalichman, Matthews, \& Adler, 2013), in our study almost 50\% of the HIV-infected group included non-Hispanic Blacks and Hispanics in comparison to $25 \%$ of the HIVuninfected group. These differences support that interventions, including HIV and substance use prevention, need to be specifically tailored for affected populations. Although the association between HIV and drug use is higher in males than females, it still remains elevated for women with HIV compared to women without HIV. Globally, female HIV-infected drug users may not regularly engage in HIV care and receive the benefits of ART and specific implementation approaches are needed that consider structural and contextual level barriers to care (Metsch et al., 2015). While drug use was more prevalent in all age groups comparing HIV-infected individuals to uninfected individuals, young adults (age 18-25) with HIV are of particular concern. More than $60 \%$ of HIV-infected young adults in our study used illegal drugs in the past year. Young adults are undergoing many important neurodevelopmental, psychosocial, and lifestyle changes and are more prone to engaging in risky behaviors as compared to older age groups. This can lead to challenges with adherence as they age. Drug use can further disrupt this period of change (Chambers, Taylor, \& Potenza, 2003; Degenhardt, Stockings, Patton, Hall, \& Lynskey, 2016; Squeglia \& Gray, 2016) and lead to unexpected complications in this population sub-group.

Although a major strength of this study is the nationally representative data source which allows us to compare an HIV-infected group to an HIV-uninfected group, our study has several limitations. Our estimate of HIV prevalence of $0.19 \%$ using combined 2015 to 2014 data from NSDUH among adults 18 or older is slightly lower than other national estimates by the Centers for Disease Control (prevalence of $0.295 \%$ of people ages 13 and older living with diagnosed HIV infection in the United States) (Hall et al., 2015). This may be due to the nature of the self-reported HIV question which asked if the participant had been told by a doctor or other health care professional that they had HIV/ AIDS. HIV-infected individuals misclassified as negative because they are unaware of their infection status may be among the individuals at greatest risk for not seeking care and receiving HIV testing and have higher alcohol and drug use levels. The exclusion of incarcerated, institutionalized, and homeless adults from NSDUH may also contribute to the underestimated HIV prevalence and limit the generalizability of our study.

Findings from this study help us understand the highly interconnected nature of drug use and HIV at the population level and have important implications with respect to HIV prevention, substance use prevention and treatment, as well as HIV care and treatment. Public health efforts are needed to screen and assess those infected with HIV first for substance use to prevent progression to abuse/dependence, then for substance dependence or abuse in order to link those in need of assistance to care and treatment. For uninfected individuals that engage in high-risk behaviors, the risk of HIV acquisition via needle exposure or sexual exposure can be reduced through pre-exposure prophylaxis (Landovitz, 2015) as well as other harm-reduction strategies, though the effectiveness of pre-exposure prophylaxis highly depends on adherence (Grant et al., 2010). A reduction in drug use may have important implications for the health of people living with HIV. 


\section{Role of funding sources}

This study was partially funded by the National Institutes of Health, National Institute on Drug Abuse (NIH-NIDA) R01 DA037866 (PI: Martins).

\section{Contributors}

The authors of this study are: Ms. Stephanie Shiau (SS), Dr. Stephen M. Arpadi (SMA), Dr. Michael T. Yin (MTY), and Dr. Silvia S. Martins (SSM, corresponding author). SSM and SS designed and conceptualized the study. SS analyzed the data under the supervision of SSM. SS wrote the first full draft of the manuscript. All authors critically reviewed and edited the manuscript, and approved the final version for submission (SS, SMA, MTY, SSM).

\section{Conflict of interest}

The authors have no conflict of interest to declare

\section{Acknowledgments}

The authors would like to thank the National Survey on Drug Use and Health NSDUH study respondents and Substance Abuse and Mental Health Service Administration (SAMHSA) for access to the NSDUH files.

\section{References}

American Psychiatric Association (2000). Diagnostic and statistical manual of mental disorders (4th ed.) (text rev).

Arnsten, J. H., Demas, P. A., Grant, R. W., Gourevitch, M. N., Farzadegan, H., Howard, A. A. et al. (2002). Impact of active drug use on antiretroviral therapy adherence and viral suppression in HIV-infected drug users. Journal of General Internal Medicine, 17, 377-381.

Attia, S., Egger, M., Muller, M., Zwahlen, M., \& Low, N. (2009). Sexual transmission of HIV according to viral load and antiretroviral therapy: Systematic review and meta-analysis. AIDS, 23, 1397-1404.

Azar, M. M., Springer, S. A., Meyer, J. P., \& Altice, F. L. (2010). A systematic review of the impact of alcohol use disorders on HIV treatment outcomes, adherence to antiretroviral therapy and health care utilization. Drug and Alcohol Dependence, 112, 178-193.

Baggaley, R. F., Boily, M. C., White, R. G., \& Alary, M. (2006). Risk of HIV-1 transmission for parenteral exposure and blood transfusion: A systematic review and meta-analysis. AIDS, 20, 805-812.

Centers for Disease Control and Prevention (2014). HIV surveillance report - Diagnoses of HIV infection in the United States and dependent areas.

Centers for Disease Control and Prevention (2015). HIV and injection drug use in the United States.

Chambers, R. A., Taylor, J. R., \& Potenza, M. N. (2003). Developmental neurocircuitry of motivation in adolescence: A critical period of addiction vulnerability. The American Journal of Psychiatry, 160, 1041-1052.

Conrad, C., Bradley, H. M., Broz, D., Buddha, S., Chapman, E. L., Galang, R. R., et al. (2015) Community outbreak of HIV infection linked to injection drug use of oxymorphoneIndiana, 2015. MMWR. Morbidity and Mortality Weekly Report, 64, 443-444.

Cropsey, K. L., Willig, J. H., Mugavero, M. J., Crane, H. M., McCullumsmith, C., Lawrence, S. et al. (2016). Cigarette smokers are less likely to have undetectable viral loads: Results from four HIV clinics. Journal of Addiction Medicine, 10, 13-19.

Degenhardt, L., Stockings, E., Patton, G., Hall, W. D., \& Lynskey, M. (2016). The increasing global health priority of substance use in young people. The Lancet Psychiatry, 3 , 251-264.

Galvan, F. H., Bing, E. G., Fleishman, J. A., London, A. S., Caetano, R., Burnam, M. A., et al. (2002). The prevalence of alcohol consumption and heavy drinking among people with HIV in the United States: Results from the HIV cost and services utilization study. Journal of Studies on Alcohol, 63, 179-186.

Grant, R. M., Lama, J. R., Anderson, P. L., McMahan, V., Liu, A. Y., Vargas, L., et al. (2010). Preexposure chemoprophylaxis for HIV prevention in men who have sex with men. The New England Journal of Medicine, 363, 2587-2599.

Gray, R. H., Wawer, M. J., Brookmeyer, R., Sewankambo, N. K., Serwadda, D., WabwireMangen, F., et al. (2001). Probability of HIV-1 transmission per coital act in monogamous, heterosexual, HIV-1-discordant couples in Rakai, Uganda. Lancet, 357, 1149-1153.

Hall, H. I., An, Q., Tang, T., Song, R., Chen, M., Green, T., et al. (2015). Prevalence of diagnosed and undiagnosed HIV infection-United States, 2008-2012. MMWR. Morbidity and Mortality Weekly Report, 64, 657-662.
Helleberg, M., Afzal, S., Kronborg, G., Larsen, C. S., Pedersen, G., Pedersen, C., et al. (2013) Mortality attributable to smoking among HIV-1-infected individuals: A nationwide, population-based cohort study. Clinical Infectious Diseases, 56, 727-734.

Hinkin, C. H., Barclay, T. R., Castellon, S. A., Levine, A. J., Durvasula, R. S., Marion, S. D., et al. (2007). Drug use and medication adherence among HIV-1 infected individuals. AIDS and Behavior, 11, 185-194.

Hudgins, R., McCusker, J., \& Stoddard, A. (1995). Cocaine use and risky injection and sexual behaviors. Drug and Alcohol Dependence, 37, 7-14.

Justice, A. C., McGinnis, K. A., Tate, J. P., Braithwaite, R. S., Bryant, K. J., Cook, R. L., et al. (2016). Risk of mortality and physiologic injury evident with lower alcohol exposure among HIV infected compared with uninfected men. Drug and Alcohol Dependence, 161, 95-103.

Kapadia, F., Vlahov, D., Donahoe, R. M., \& Friedland, G. (2005). The role of substance abuse in HIV disease progression: Reconciling differences from laboratory and epidemiologic investigations. Clinical Infectious Diseases, 41, 1027-1034.

Korthuis, P. T., Zephyrin, L. C., Fleishman, J. A., Saha, S., Josephs, J. S., McGrath, M. M., et al. (2008). Health-related quality of life in HIV-infected patients: The role of substance use. AIDS Patient Care and STDs, 22, 859-867.

Landovitz, R. J. (2015). Preexposure prophylaxis for HIV prevention: What we know and what we still need to know for implementation. Topics in Antiviral Medicine, 23 85-90.

Marcus, J. L, Chao, C. R, Leyden, W. A., Xu, L, Quesenberry, C. P. Jr, Klein, D. B., et al. (2016). Narrowing the gap in life expectancy between HIV-infected and HIV-uninfected individuals with access to care. Journal of Acquired Immune Deficiency Syndromes.

Massanella, M., Gianella, S., Schrier, R., Dan, J. M., Perez-Santiago, J., Oliveira, M. F., et al. (2015). Methamphetamine use in HIV-infected individuals affects T-cell function and viral outcome during suppressive antiretroviral therapy. Scientific Reports, 5 13179.

McCance-Katz, E. F. (2005). Treatment of opioid dependence and coinfection with HIV and hepatitis $C$ virus in opioid-dependent patients: The importance of drug interactions between opioids and antiretroviral agents. Clinical Infectious Diseases, 41(Suppl. 1), S89-S95.

Metsch, L., Philbin, M. M., Parish, C., Shiu, K., Frimpong, J. A., \& Giang le, M. (2015). HIV testing, care, and treatment among women who use drugs from a global perspective: Progress and challenges. Journal of Acquired Immune Deficiency Syndromes, 69(Suppl. 2), S162-S168.

Molitor, F., Truax, S. R., Ruiz, J. D., \& Sun, R. K. (1998). Association of methamphetamine use during sex with risky sexual behaviors and HIV infection among non-injection drug users. The Western Journal of Medicine, 168, 93-97.

Nolan, S., Milloy, M. J., Zhang, R., Kerr, T., Hogg, R. S., Montaner, J. S., et al. (2011). Adherence and plasma HIV RNA response to antiretroviral therapy among HIV-seropositive injection drug users in a Canadian setting. AIDS Care, 23, 980-987.

Ompad, D. C., Giobazolia, T. T., Barton, S. C., Halkitis, S. N., Boone, C. A., Halkitis, P. N., et al (2016). Drug use among HIV + adults aged 50 and older: Findings from the GOLD Il study. AIDS Care, 1-5.

Pellowski, J. A., Kalichman, S. C., Matthews, K. A., \& Adler, N. (2013). A pandemic of the poor: Social disadvantage and the U.S. HIV epidemic. The American Psychologist, 68 197-209.

Purcell, D. W., Parsons, J. T., Halkitis, P. N., Mizuno, Y., \& Woods, W. J. (2001). Substance use and sexual transmission risk behavior of HIV-positive men who have sex with men. Journal of Substance Abuse, 13, 185-200.

Samji, H., Cescon, A., Hogg, R. S., Modur, S. P., Althoff, K. N., Buchacz, K., et al. (2013). Closing the gap: Increases in life expectancy among treated HIV-positive individuals in the United States and Canada. PloS One, 8, e81355.

Spiller, M. W., Broz, D., Wejnert, C., Nerlander, L., \& Paz-Bailey, G. (2015). HIV infection and HIV-associated behaviors among persons who inject drugs-20 cities, United States, 2012. MMWR. Morbidity and Mortality Weekly Report, 64, 270-275.

Squeglia, L. M., \& Gray, K. M. (2016). Alcohol and drug use and the developing brain. Current Psychiatry Reports, 18, 46.

Strathdee, S. A., \& Stockman, J. K. (2010). Epidemiology of HIV among injecting and noninjecting drug users: Current trends and implications for interventions. Current HIV/ AIDS Reports, 7, 99-106.

Substance Abuse and Mental Health Services Administration (2012). National Survey on drug use and health sample design report.

Substance Abuse and Mental Health Services Administration (2005-2014). National Survey on drug use and health. Public use data files. 